\title{
The impact of initial antibiotic therapy (linezolid, vancomycin, daptomycin) on hospital length of stay for complicated skin and soft tissue infections
}

Ewa Szczypinska ${ }^{1}$, Alexander Velazquez ${ }^{1}$, Diana Salazar ${ }^{1}, C^{\prime}$ Andrew DeRyke ${ }^{1}$, Beata Raczynski ${ }^{1}$ and Mark R Wallace ${ }^{1,2^{*}}$

\begin{abstract}
Background: Empiric therapy of inpatient skin and soft tissue infections (SSTIs) generally require methicillin resistant Staphylococcus aureus (MRSA) coverage. Limited data are available to directly compare the effect of initial antibiotic choice on treatment outcomes and length of stay (LOS).

Objective: To assess potential differences in length of hospital stay when inpatients with complex skin and soft tissue infections (SSTIS) were initially treated with either vancomycin, linezolid, or daptomycin.

Methods: A retrospective review of 219 patients diagnosed with inpatient SSTI who received linezolid, vancomycin, or daptomycin for $>48$ hours was performed. Data collected included demographics, comorbidities, microbiologic/ laboratory data, additional management (surgical, non-study antibiotics), hospital LOS, treatment outcome and morbidity/mortality.
\end{abstract}

Results: The three groups evaluated were linezolid $(n=45)$, vancomycin $(n=90)$ daptomycin $(n=84)$. There was no difference between the three groups with respect to gender, age, comorbidities, leukocytosis, fever, antibiotics prior to admission, site of infection culture results and surgical intervention. One death was recorded, not associated with diagnosis of SSTI. No significant difference in LOS was found $(P=0.525)$ between the 3 groups. The mean LOS in entire cohort was 4.5 days (SD \pm 2.5$)$; thirty patients had prolonged LOS for non-SSTI reasons; reanalyzing the data without these 30 patients did not produce any difference in the mean LOS between the 3 groups. Switching vancomycin just prior to discharge to facilitate outpatient therapy was common but did not impact LOS.

Conclusions: No difference was detected in hospital length of stay with respect to the initial choice of antibiotic (linezolid, vancomycin, or daptomycin) for SSTI. The three antibiotic regimens were equally effective in treating SSTIs as judged by LOS, irrespective of age, gender, comorbidities or baseline severity of SSTI. Given the large standard deviation in LOS, this result should be confirmed by larger studies.

Keywords: Vancomycin; Daptomycin; Linezolid; Skin and soft tissue infections

\footnotetext{
* Correspondence: mrwallace1@yahoo.com

'Department of Infectious Disease, Orlando Health, Orlando, FL, USA

${ }^{2} 21$ W Columbia St., Suite 102, Orlando, FL 32806, USA
} 


\section{Background}

Skin and soft tissue infections (SSTIs) account for a large proportion of hospitalizations. In 2004, approximately 870,000 U.S. hospital admissions were due to SSTIs, an increase of $29 \%$ over 2000 data (Edelsbert et al. 2009). Though multiple studies have found varying average inpatient stays and costs for SSTIs, a recent analysis found an average stay of 6.1 days and cost 6830 United States dollars (USD) per episode (Menzin et al. 2010).

The most common organisms involved in SSTIs are Staphylococcus aureus and B-haemolytic streptococci (Rajan 2012). The initial treatment regimen is based on clinical presentation, microbiologic data, hospital antibiogram, physician's discretion and the pharmacy formulary. Given growing antibiotic resistance, empiric antimicrobial therapies for severe SSTIs must now have MRSA activity, as about half of S. aureus infections are methicillin resistant (Menzin et al. 2010; Rajan 2012). Currently, the leading options for empiric inpatient coverage for SSTIs are linezolid, vancomycin, and daptomycin (Bounthavong et al. 2011). Ceftaroline, telavancin, and tigecycline are newer, but less used, options in this category (van Hal and Paterson 2011).

Daptomycin has superior in vitro bactericidal activity against MRSA when compared with vancomycin and linezolid (Maraconescu et al. 2012). Multiple analyses have compared vancomycin (the incumbent "gold standard") to linezolid and daptomycin. Though "suggestions" of superiority for daptomycin and/or linezolid have been found (van Hal and Paterson 2011; Bounthavong and Hsu 2012; Logman et al. 2010; Davis et al. 2007), the actual value with respect to hospital LOS from changing from initial therapy with vancomycin, a time tested and inexpensive antibiotic, to one of the more expensive newer agents as initial therapy for inpatient SSTIs have not been convincingly demonstrated. As part of a cost benefit analysis at our medical center, we conducted a retrospective study to evaluate the impact of initial inpatient antibiotic choice (daptomycin, linezolid, or vancomycin) on SSTI length of hospital stay.

\section{Methods}

A retrospective cohort review was performed on Orlando Health inpatients that were diagnosed with a skin or soft tissue infection and received one of the three study antibiotics (daptomycin, linezolid, or vancomycin) between January 2009 and September 2010. Charts were selected by ICD-9 codes (680.0 - 686.9) and comprehensively reviewed. One dose of a non-study antibiotic was permitted prior to initiation of therapy with vancomycin, linezolid or daptomycin.

\section{Inclusion criteria}

Patients between the ages of $18-85$ with an acute SSTI, defined as three or more of the following: warmth, erythema, swelling, pain, tenderness, lymph node swelling/ tenderness, drainage/discharge, or induration, for less than two weeks. Patients were also included if they had an abscess requiring incision and drainage at bedside or in the operating room. Initial antibiotic treatment was daptomycin, linezolid (intravenous or oral), or vancomycin and continued for at least 48 hours. The choice of initial antibiotic was entirely dependent on the attending physician's preference.

\section{Exclusion criteria}

Patients were excluded if they had osteomyelitis (suspected or proven), decubitus ulcer, necrotizing fasciitis, myositis, gas gangrene, a Gram positive isolate proven resistant to one or more study antibiotics, Gram negative infection, or the presence of concomitant infection upon admission (i.e. pneumonia, UTI).

\section{Data collection, definitions, statistics}

Hospital length of stay was evaluated in relation to the specific antimicrobial regimen chosen on admission. Length of stay was calculated based on nights spent in the hospital. A prolonged stay occurred when patients were kept in hospital beyond the requisite stay for SSTI treatment for unrelated medical or social problems.

Additional data collected included demographics (age, gender), clinical presentation (collected from physician's progress notes, including antibiotics prior to admission, anatomical site of infection, vital signs, duration of symptoms), co-morbidities (HIV status, diabetes mellitus (DM), peripheral vascular disease (PVD), end stage renal disease (ESRD), immunosuppressive therapy, malignancy), social history (tobacco use), and laboratory/radiological data (white blood cell count, microbiological data, creatinine, imaging). Treatment data collected included surgical/bedside interventions performed, intensive care unit admission, additional non-study antibiotics administered, and whether a switch in therapy occurred. Switching of antibiotic was defined as a switch from study antibiotic to a different study antibiotic or another non-study antibiotic during the hospitalization. Switching of antibiotics was classified into one of the following categories:

1. Transition to outpatient therapy: (changing to oral or simplified once daily intravenous therapy).

2. Treatment failure. No clinical improvement after $\geq 48$ hours; switched to a different antibiotic based on physician's judgment.

3. De-escalation to a B-lactam antibiotic after organism identification and susceptibility available 
(i.e. methicillin susceptible Staphylococcusaureus or Group A streptococci).

4. Allergy or adverse reaction.

5. Therapeutic preference of the attending physician.

Descriptive statistics for all data were collected and stored using Microsoft Excel software. Statistical tests were performed using SPSS software with a p value $<0.05$ used to define statistical significance. Nominal data were compared by using the Pearson $X^{2}$ test. Age and length of stay measures were analyzed using an ANOVA.

This research was approved through the Institutional Review Board (IRB) at Orlando Health.

\section{Results}

Four hundred and seventy two charts were reviewed; a total of 219 patients met the inclusion criteria (daptomycin $n=84$, linezolid $n=45$, and vancomycin $n=90$ ). No difference in hospital length of stay was observed regardless of initial antibiotic choice for patients admitted to the hospital with skin and soft tissue infection $(\mathrm{p}=0.53)$ (Figure 1). The mean LOS in entire cohort was 4.5 days $(\mathrm{SD} \pm 2.5)$. Thirty patients had a prolonged hospital length of stay beyond what was required for treatment of their SSTI; the data with these patients excluded also showed no difference in LOS: $(n=189$, $\mathrm{p}=0.49)$ (Figure 2).

The mean age in the daptomycin, linezolid and vancomycin groups was 49,48 , and 52 years $(\mathrm{SD}+/-2.5)$, respectively. There was no difference in gender $(\mathrm{p}=0.64)$, history of intravenous drug $(\mathrm{p}=0.90)$ or tobacco use $(\mathrm{p}=0.26)$. The clinical presentation was not significantly different between the three antibiotic groups when evaluated by preadmission antibiotics, site of infection culture results, adenopathy, fever, leukocytosis, drainable foci, radiological evidence of infection, or requirement for surgical intervention (Table 1). Furthermore, except for end stage renal failure which was over represented in the daptomycin group $(\mathrm{p}=0.038)$, there was no difference between observed co-morbidities including

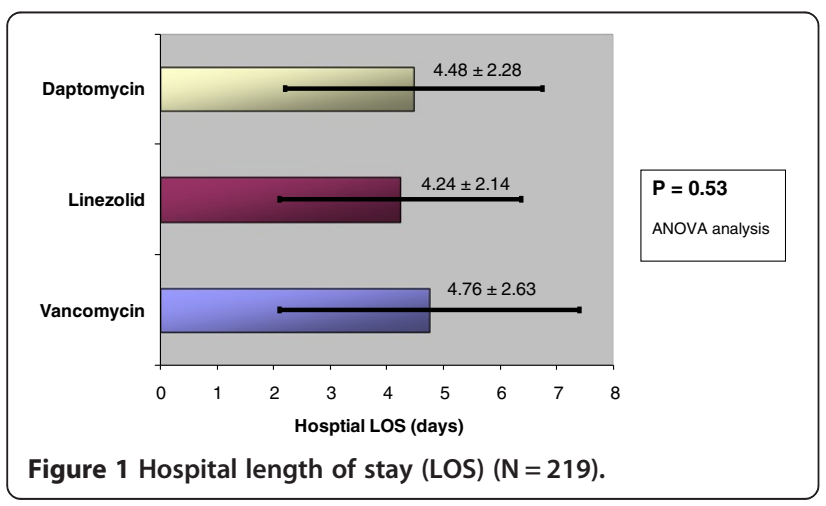

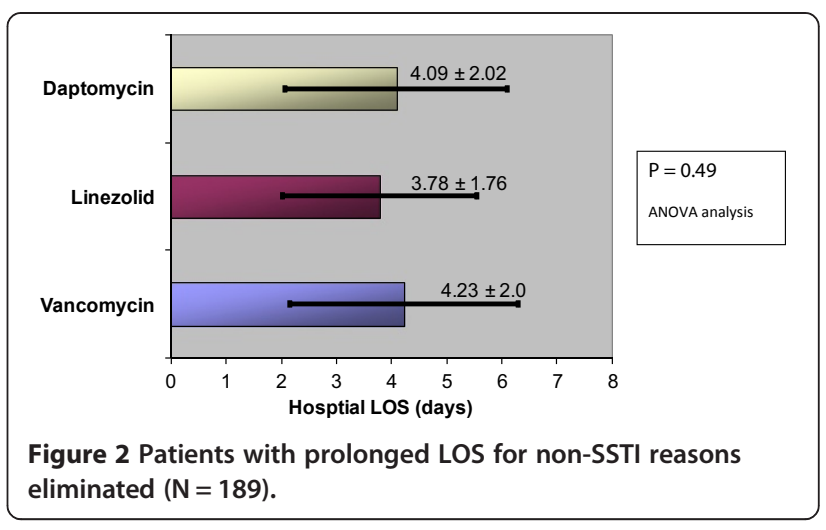

diabetes, heart failure, immunosuppressive drugs, HIV, peripheral vascular disease, history of malignancy, history of transplant, or previous known colonization with MRSA (Table 2). Vancomycin levels were collected in 44 cases $(49 \%)$, with trough levels $>/=10 \mathrm{mg} / \mathrm{L}$ in $87 \%$ cases $(\mathrm{n}=38)$.

There was a significant difference in switching of antibiotics during treatment. The vancomycin group had the highest percentage of switching (55\%), followed by daptomycin $(19 \%)$, and linezolid $(11 \%)(\mathrm{p}<0.05)$. The most common reason for switching antibiotics in all groups was a transition to outpatient therapy; $88 \%$ of vancomycin changes were to either daptomycin, so as to facilitate once daily outpatient intravenous antibiotic therapy, or a transition to oral treatment. The vancomycin patients changed to daptomycin almost invariably occurred on the last hospital day and had no influence on LOS. Only one patient in any group (daptomycin) was changed

Table 1 Clinical and microbiologic features of SSTIs

\begin{tabular}{lcccc}
\hline & Daptomycin & Linezolid & Vancomycin & P value \\
\hline $\begin{array}{l}\text { Antibiotics prior } \\
\text { to admission }\end{array}$ & $29(34)$ & $11(24)$ & $20(22)$ & 0.16 \\
Adenopathy & $6(7)$ & $0(0)$ & $9(10)$ & 0.09 \\
Fever & $14(16)$ & $7(15)$ & $20(22)$ & 0.53 \\
Leukocytosis & $53(63)$ & $20(44)$ & $46(51)$ & 0.09 \\
Drainable Foci & $34(40)$ & $16(35)$ & $38(42)$ & 0.75 \\
$\begin{array}{l}\text { Coadministration } \\
\text { non-study antibiotic }\end{array}$ & $64(76)$ & $32(71)$ & $76(84)$ & 0.16 \\
$\begin{array}{l}\text { Radiological evidence } \\
\text { of collection/abscess }\end{array}$ & $10(11)$ & $2(4)$ & $12(13)$ & 0.27 \\
Wound + MRSA & $26(31)$ & $13(29)$ & $28(31)$ & \\
Wound + MSSA & $7(8)$ & $7(16)$ & $7(8)$ & \\
$\begin{array}{l}\text { Positive blood } \\
\text { cultures* }\end{array}$ & $2(2)$ & 0 & $4(4)$ & \\
\hline
\end{tabular}

*All positive blood cultures were MRSA.

n (\%).

Pearson $X^{2}$ test analysis. 
Table 2 Comorbidities of SSTI patients

\begin{tabular}{lcccc}
\hline & Daptomycin & Linezolid & Vancomycin & P value \\
\hline Diabetes & $24(28)$ & $9(20)$ & $23(25)$ & 0.56 \\
Heart failure & $1(1)$ & $3(6)$ & $3(3)$ & 0.24 \\
ESRD & $7(8)$ & $0(0)$ & $2(2)$ & 0.03 \\
Immunosuppressive & $4(4)$ & $1(2)$ & $5(5)$ & 0.67 \\
therapy & & & & \\
HIV & $1(1)$ & $3(6)$ & $6(6)$ & 0.16 \\
$\begin{array}{l}\text { Peripheral vascular } \\
\text { disease }\end{array}$ & $6(7)$ & $3(6)$ & $4(4)$ & 0.73 \\
History malignancy & $5(6)$ & $1(2)$ & $11(12)$ & 0.09 \\
History transplant & $1(1)$ & $1(2)$ & $3(3)$ & 0.63 \\
Colonized MRSA & $9(10)$ & $5(11)$ & $9(10)$ & 0.97 \\
\hline $\mathrm{n}$ (\%). & & & & \\
Pearson $X^{2}$ test analysis. & & &
\end{tabular}

due to treatment failure (Table 3). No patient was excluded or switched because of Gram positive resistance.

One death was recorded, not associated with the diagnosis of SSTI.

\section{Discussion}

This retrospective "real-world" study found no significant differences in length of stay between the three most commonly used empiric inpatient anti-MRSA antibiotics for skin and soft tissue infections requiring inpatient care. Previous in vitro work supports daptomycin as the superior agent (Maraconescu et al. 2012). Multiple clinical studies, analyses and reviews, have suggested a possible advantage for linezolid (Menzin et al. 2010; Bounthavong et al. 2011; van $\mathrm{Hal}$ and Paterson 2011; Bounthavong and Hsu 2012; Logman et al. 2010; Itani et al. 2012; Barron et al. 2012; Watkins et al. 2012) or daptomycin (Itani et al. 2010; Quist et al. 2012; Falcone et al. 2012; Bliziotis et al. 2010; Davis et al. 2007) as compared to vancomycin in terms of time to clinical cure, hospital length of stay, or both. Despite the evidence favoring linezolid or daptomycin over vancomycin, some authors have been unconvinced of the clinical relevance of the purported superiority of the newer agents (Eckmann and Dryden 2010). We chose to

Table 3 Reason antibiotics switched during treatment

\begin{tabular}{lccc}
\hline & $\begin{array}{c}\text { Daptomycin } \\
\text { (16) }\end{array}$ & $\begin{array}{c}\text { Linezolid } \\
\text { (5) }\end{array}$ & $\begin{array}{c}\text { Vancomycin } \\
\mathbf{( 5 0 )}\end{array}$ \\
\hline De-escalation & 3 & 1 & 4 \\
Transition to outpatient & 11 & 2 & 44 \\
Presumed treatment failure & 1 & 0 & 0 \\
Allergy or adverse effect & 0 & 1 & 1 \\
Therapeutic preference & 1 & 1 & 1 \\
\hline
\end{tabular}

focus on LOS as a single key parameter of efficacy as it is closely tied to hospital cost and is more straightforward than assessment of clinical parameters such as time of resolution of cellulitis, etc.

Vancomycin's main advantages are its 50 year track record and low cost, but its use requires careful monitoring to avoid nephrotoxicity (van Hal and Paterson 2011). Though overt resistance remains rare, some institutions have noted "MIC Creep," a situation in which vancomycin MICs $\geq 1.5 \mathrm{mg} / \mathrm{l}$ are found with increasing frequency. Such isolates may be difficult to cure with vancomycin, even at higher doses (Brink 2012). At our institution, such isolates comprise $<1.5 \%$ of all Staphylococcus aureus isolates using the Vitek 2 methodology (BioMerieux). Daptomycin is a remarkably easy drug to use, but it may occasionally cause myopathy or serious eosinophilic pneumonia (van Hal and Paterson 2011). A larger concern is the increasing frequency of daptomycin resistance (van $\mathrm{Hal}$ and Paterson 2011; van Hal et al. 2011; Velazquez et al. 2013). Linezolid is the simplest of the three antibiotics, given its high oral bioavailability and simple intravenous to oral conversion, but its use may be complicated by thrombocytopenia, peripheral neuropathy and drug interactions with selective serotonin inhibitors (Rajan 2012). Resistance to linezolid remains rare, but may be increasing (van Hal and Paterson 2011).

This retrospective study has limitations. The patients were allocated into groups by the preferences of the attending physicians, many of whom held strong views on the relative superiority of one agent vs. the others. Attendings may have started "sicker" patients on the nonvancomycin regimens, though we have no evidence this occurred as the groups were comparable with respect to demographics, admission WBC, fever and baseline comorbidities. Institutions with diminishing vancomycin susceptibility may experience poorer outcomes with vancomycin use. The study may have been underpowered to detect differences; larger numbers might have turned the trend in favor of linezolid into a statistically significant finding.

Vancomycin remains a key antibiotic in the gram positive armamentarium. We found no evidence for any significant difference in length of stay for SSTI between the three common initially used inpatient agents, and are continuing to advise clinicians at our medical center to start with vancomycin for complicated inpatients SSTIs, (unless there were contraindications), both to limit cost and the risk of daptomycin or linezolid resistance. How long vancomycin will remain the workhorse inpatient choice remains unclear, and will likely depend on differential resistance among these 3 agents, new drugs such as ceftaroline, and external economic factors. 


\section{Competing interest}

The authors declare that they have no competing interest.

\section{Authors' contributions}

All authors were involved in chart review and manuscript drafting. ES, CAD and MRW designed the study. All authors read and approved the final manuscript.

\section{Acknowledgement}

Presented as a poster at the 2011 Infectious Disease Society of America Meeting.

Received: 9 July 2013 Accepted: 18 December 2013

Published: 30 December 2013

\section{References}

Barron J, Turner R, Jaejer M, Adamson W, Singer J (2012) Comparing the use of intravenous antibiotics under the medical benefit with the use of oral antibiotics under the pharmacy benefit in treating skin and soft tissue infections. Manag Care 21:44-52

Bliziotis IA, Plessa E, Peppas G, Falagas ME (2010) Daptomycin versus other antimicrobial agents for the treatment of skin and soft tissue infections: a meta- analysis. Ann Pharmacother 44:97-106

Bounthavong M, Hsu DI (2012) Cost-effectiveness of linezolid in methicillinresistant Staphylococcus aureus skin and skin structure infections. Expert Rev Pharmacoecon Outcomes Res 12:683-698

Bounthavong M, Zargarzadeh A, Hsu DI (2011) Cost effectiveness analysis of Linezolid, Daptomycin, and Vancomycin in Methicillin-resistant Staphylococcus aureus: complicated skin and skin structure infection using Bayesian methods of evidence. Value Health 14:631-639

Brink AJ (2012) Does resistance in severe infections caused by methicillinresistant Staphylococcus aureus give you the 'creeps'? Curr Opin Crit Care 18:451-459

Davis SL, McKinnon PS, Hall LM et al (2007) Daptomycin versus vancomycin for complicated skin and skin structure infections: clinical and economic outcomes. Pharmacotherapy 27:1611-1618

Eckmann C, Dryden M (2010) Treatment of complicated skin and soft-tissue infections caused by resistant bacteria: value of linezolid, tigecycline, daptomycin and vancomycin. Eur J Med Res 15:554-563

Edelsbert J, Taneja C, Zervos M et al (2009) Trends in US hospital admissions for skin and soft tissue infections. Emerg Infect Dis 15:1516-1518

Falcone M, Russo A, Popmep ME, Vena A et al (2012) Retrospective case-control analysis of patients with staphylococcal infections receiving daptomycin or glycopeptides therapy. Int J Antimicrob Agents 39:64-68

Itani KM, Dryden MS, Bhattacharyya H, Kunkel MJ, Baruch AM, Weigelt JA (2010) Efficacy and safety of linezolid versus vancomycin for the treatment of complicated skin and soft-tissue infections proven to be caused by methicillin-resistant Staphylococcus aureus. Am J Surg 199:804-816

Itani KM, Biswas P, Reisman A, Bhattacharyya H, Baruch AM (2012) Clinical efficacy of oral linezolid compared with intravenous vancomycin for the treatment of methicillin-resistant Staphylococcusaureus-complicated skin and soft tissue infections: a retrospective, propensity score-matched case-control analysis. Clin Ther 34:1667-1673

Logman JF, Stephens J, Heeg B et al (2010) Cost effectiveness of antibiotics for the treatment of MRSA complicated skin and soft tissue infections. Curr Med Res Opin 26:1565-1578

Maraconescu P, Graviss EA, Musher DM (2012) Rates of killing of methicillin resistant Stapylococcus aureus by ceftaroline, daptomycin, telavancin compared. Scand J Infect Dis 44:884

Menzin J, Marton JP, Merers JL et al (2010) Inpatient treatment patterns, outcomes, and costs of skin and skin structure infections because of Staphylococcus aureus. Am J Infect Control 38:44-49

Quist SR, Fierlbeck G, Seaton RA, Loeffler J, Chaves RL (2012) Comparative randomized clinical trial against glycopeptides supports the use of daptomycin as first-line treatment of complicated skin and soft-tissue infections. Int J Antimicrob Agents 39:90-91

Rajan S (2012) Skin and soft-tissue infections: classifying and treating a spectrum. Cleve Clin J Med 79:57-66

van Hal SJ, Paterson DL (2011) New gram-positive antibiotics: better than vancomycin? Curr Opin Infect Dis 24:515-520 van Hal SJ, Paterson DL, Gosbell IB (2011) Emergence of daptomycin resistance following vancomycin-unresponsive Staphylococcus aureusbacteraemia in a daptomycin-naïve patient - a review of the literature. Eur J Clin Microbiol Infect Dis 30:603-610

Velazquez A, DeRyke CA, Goering R, Hoover V, Wallace M (2013) Daptomycin non- susceptible Staphylococcus aureus at a US medical center. Clin Microbiol Infect, Accepted for publication, doi: 10.1111/1469-0691.12171

Watkins RR, Lemonovich TL, Thomas MF (2012) An evidence-based review of linezolid for the treatment of methicillin-resistant Staphylococcus aureus (MRSA): place in therapy. Core Evidence 7:131-143

\section{doi:10.1186/2193-1801-2-696}

Cite this article as: Szczypinska et al.: The impact of initial antibiotic therapy (linezolid, vancomycin, daptomycin) on hospital length of stay for complicated skin and soft tissue infections. SpringerPlus 2013 2:696.

\section{Submit your manuscript to a SpringerOpen ${ }^{\odot}$ journal and benefit from:}

- Convenient online submission

- Rigorous peer review

- Immediate publication on acceptance

- Open access: articles freely available online

- High visibility within the field

- Retaining the copyright to your article

Submit your next manuscript at $>$ springeropen.com 